\title{
Correspondence
}

\section{Clarify the limits of climate models}

Mark Maslin and Patrick Austin suggest that scientists should explain the statistical uncertainties in models of climate-change impacts (Nature 486, 183-184; 2012), but these models are tools for insight, not prediction. A diversity of models benefits both science and science-based policy guidance.

We believe that future models will be more informative than those of today. The public-image problem of current models stems partly from scientists' failures to identify the limitations openly. It is important to distinguish between questions for which current models are useful as prediction engines and those for which the models merely probe possibilities. The role of science is to reflect on the plausibility and relevance of such possibilities.

Deep uncertainty is not foreign to policy-makers, who often have to weigh the advantages of deciding to wait against the potentially high costs of waiting. David A. Stainforth, Leonard A. Smith London School of Economics, UK. d.a.stainforth@lse.ac.uk

\section{ENCODE leads the way on big data}

The ENCODE project offers a fresh perspective on big data by providing an organized framework for genomics (www. nature.com/encode). Other big-data efforts tend to focus on rapidly locating needles in petabyte-sized haystacks (such as finding the Higgs boson, for instance), whereas ENCODE aims to supply a structured overview.

ENCODE's organization of information is hierarchical, with raw data at the bottom and layers of annotation above. The processed summaries become progressively broader - for example, starting at the level of signals representing the degree to which DNA is bound by transcription factors, moving on to the locations of sites where these factors bind, and then to overviews of regulatory networks. At the summit are the linked publications documenting the annotation.

The ENCODE data model could be useful in other fields: for example, astronomy and Earth science are in the process of organizing their reams of data (M. J. Raddick and A.S. Szalay Science 329, 1028-1029; 2010), but don't yet compare with ENCODE in terms of the level of integration.

Mark Gerstein Yale University, New Haven, Connecticut, USA. mark.gerstein@yale.edu

\section{Animal variants in sexual behaviour}

Andrew Barron and Mark Brown suggest that journalists, by merging animal and human behaviour, promote the myth that minority sexual preferences are an 'illness' (Nature 488, 151-152; 2012). They include one of my articles among their examples - 'Bat bugs turn transsexual to avoid stabbing penises' (see go.nature.com/9iwdpb) presumably because it uses 'transsexual' to describe insect behaviour. I contend that this term is valid shorthand for what the insects are doing, as well as catching the reader's attention.

Reporting of same-sex behaviour in animals can stray into troubling territory. In one example given by Barron and Brown, The Sunday Times wrote in 2006 that a study of maleoriented behaviour in sheep "could pave the way for breeding out homosexuality in humans". However, the problem here is not the terms used to describe animals' sex lives, but the leverage of the research paper to make a political point.

Homosexual behaviour is condemned as unnatural in some places around the world. By describing the huge variation in sexual behaviour across the animal kingdom, the media may be helping to destroy forever the idea that gay, lesbian, bisexual and transgender people represent some kind of aberration.

Rowan Hooper London, UK. rowan.hooper@newscientist.com

\section{A code of conduct for European scientists}

Progress in safeguarding research integrity is lamentably slow in Europe compared with the United States (Nature 488, 7; 2012). Many European universities are still unsure of their responsibilities in policing research conduct.

Punishing misconduct makes no sense without prevention, given that one study found that up to one-third of all researchers engage in questionable research practices (D. Fanelli PLoS ONE 4, e5738; 2009). What is needed is for each research institution to provide a detailed standard of responsible research conduct, along with mandatory education, so that researchers know how to uphold this standard in day-today practice.

We suggest that a European forum should be set up to educate researchers and their mentors in all matters relating to this standard. The forum would develop educational material, train and accredit teachers and remove discrepancies between research institutions.

Every university would need to set up a 'research integrity unit' to guide and supervise its research activities, and to ensure compliance with international standards.

We urge public and private research funders to lend their support to this venture, which would help to free European research from questionable research practices and make life harder for fraudsters.

Nils Axelsen Statens Serum Institut, Copenhagen, Denmark. Xavier Bosch Department of Internal Medicine, Hospital Clinic, University of Barcelona, Spain.xavbosch@clinic.ub.es

\section{Wallace pioneered astrobiology too}

The field of astrobiology, called 'exobiology' during the space programmes of the 1960s (Nature 488, 160; 2012), was anticipated six decades earlier by Alfred Russel Wallace, better known as the co-discoverer of the principle of natural selection.

Next year is the centenary of Wallace's death, when his seminal contribution to biogeography and evolution will be celebrated. But he should also be remembered as a pioneer in astrobiology, whose hypotheses are still alive today.

Wallace introduced the concept of "astro-biology" in his popular book Man's Place in the Universe (Chapman \& Hall, 1903). He reviewed the physical conditions required for organic life in terrestrial ecosystems and concluded that Earth is the only habitable planet in the Solar System.

He later published a monograph (Is Mars Habitable? Macmillan, 1907) evaluating astronomer Percival Lowell's suggestion that Mars could be "inhabited by a race of highly intelligent beings" (see Nature 74, 587-589; 1906). After analysing what was then known about the Martian climate, temperature, possible presence of water and the 'canals' thought by Lowell to indicate intelligent life, Wallace roundly refuted this idea. U. Kutschera Institute of Biology, University of Kassel, Germany. kut@uni-kassel.de

\section{CORRECTION}

The obituary of Andrew Fielding Huxley (Nature 486,474 ; 2012) omitted to mention that Hugh Huxley and Jean Hanson also proposed a sliding-filament model of muscle contraction, based on myofibril experiments. Their work was published (Nature 173, 973; 1954) alongside A. F. Huxley's Nature paper. 